\title{
Consideration of heating source for application of active thermography to concrete structure.
}

\author{
by M. Koyama*, M. Ishikawa**, H. Kasano***
}

Meisei University, School of Science and Engineering,2-1-1, Hodokubo, Hinoshi, Tokyo, Japan , e-mail: masashi.koyama@meisei-u.ac.jp

** Tokushima University, Graduate School of Technology, Industrial and Social Sciences, 2-1Minamijousanjimacho, 770-8506, Tokushima, Japan, m.ishikawa@tokushima-u.ac.jp

* Nihon University, Department of Civil Engineering, Nakagawara, Tokusada, Tamura, Koriyama, Fukushima, 963-8642, Japan, kasano@civil.ce.nihon-u.ac.jp

\begin{abstract}
Two active thermographic inspection techniques, one using halogen lamp with light focusing setup and the other, $\mathrm{CO}_{2}$ laser heating, were compared for defect detectability in concrete structures located at a distance. Experimental results showed that inspection capabilities of both methods were similar when their heat input was similar. It was also found that the $\mathrm{CO}_{2}$ laser heating method was less susceptible to discoloration on the concrete surface in comparison with halogen lamp heating. This is considered an advantage of using the $\mathrm{CO}_{2}$ laser heating. In addition, use of phase images obtained by Fourier transformation of thermal data from both methods.
\end{abstract}

\section{Introduction}

Inspection and evaluation techniques for concrete structures are essential to maintain the safety of civil buildings and infrastructure. Concrete is generally inspected using the hammer or visual inspection. However, these methods require installing temporary scaffolds for inspection, especially when testing objects in high places (such as tunnel ceilings or substructures of high bridges). This causes inconvenience, and increases the inspection cost. In this study, we focused on the use of active thermography method to inspect concrete structures. In this method, the test object is heated using a heat source, and the surface temperature during and after heating is monitored by an infrared camera; internal defects are identified by screening non-homogeneous temperature regions in the thermal images. One of the important advantages of thermography method is that it is a non-contact inspection method. Thus, the thermography method can inspect objects located far from observers if the objects are heated remotely. In this study, two remote heating methods were examined in conjunction with active thermography technique to inspect concrete structures located at a distance. One method uses a halogen lamp with light focusing apparatus, while the other employs a laser heating system. The halogen lamp is one of frequently used sources in thermographic inspection [1, 2]. Here, we attempted to focus the light of the halogen lamp using a mirror and to heat the concrete object located at a distance. On the other hand, diffusion attenuation of the laser source is smaller than that of the lamp source which makes the former more efficient in heating objects at a distance.

\section{Experiments}

\subsection{Experimental method}

In this study, measurements were conducted to obtain equivalent heat input to the concrete surface using two types of heating methods: halogen heating and laser heating. In the test, the temperature difference of the surface temperature change in the defect part and the non-defective part was confirmed.

\subsubsection{Using a halogen lamp with light focusing apparatus}

The experimental arrangement with the halogen lamp and the concrete specimen used are shown in Figs. 1 and 2. The halogen lamp was equipped with a rotating parabolic mirror used to focus the light from the lamp and to heat the specimen located far from the source. The specimen size is $300 \times 300 \times 100 \mathrm{~mm}$, and includes four blocks of polystyrene foam as artificial defects. The defects are $10 \mathrm{~mm}$ thick, and defect depths from the heated surface $d$ are 10 and $30 \mathrm{~mm}$. The specimen was placed at a distance of $10 \mathrm{~m}$ from the halogen lamp and was heated for $125 \mathrm{~s}$ (see Fig. 1 (b)). The irradiation density on the surface of the specimen was $650 \mathrm{~W} / \mathrm{m}^{2}$, which was approximately matched to the 
heat input of the laser source described in section 2.1.2. The temperature distribution on the specimen during and after heating was monitored by an infrared camera (A315, FLIR Systems, Inc.) at sampling frequency of $1 \mathrm{~Hz}$.

(a)

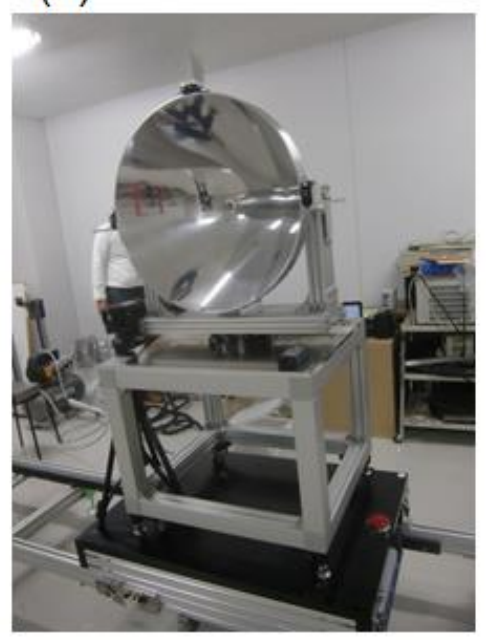

(b)

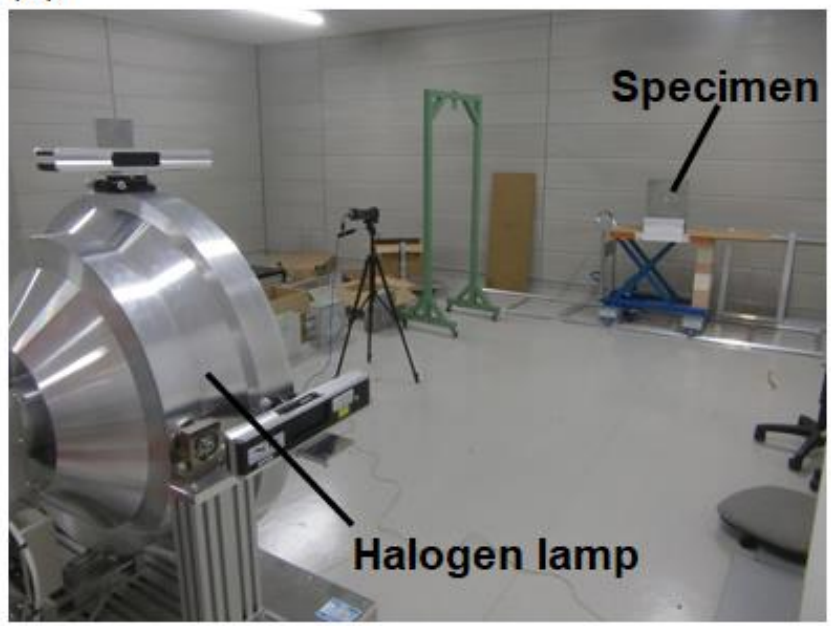

Fig. 1 (a) Halogen lamp with rotating parabolic mirror used in the experiment, and (b) Experimental setup with concrete specimen at a distance.

(a)

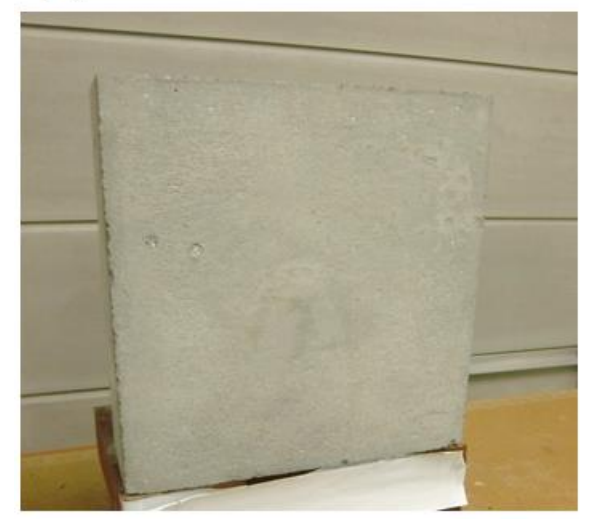

(b)

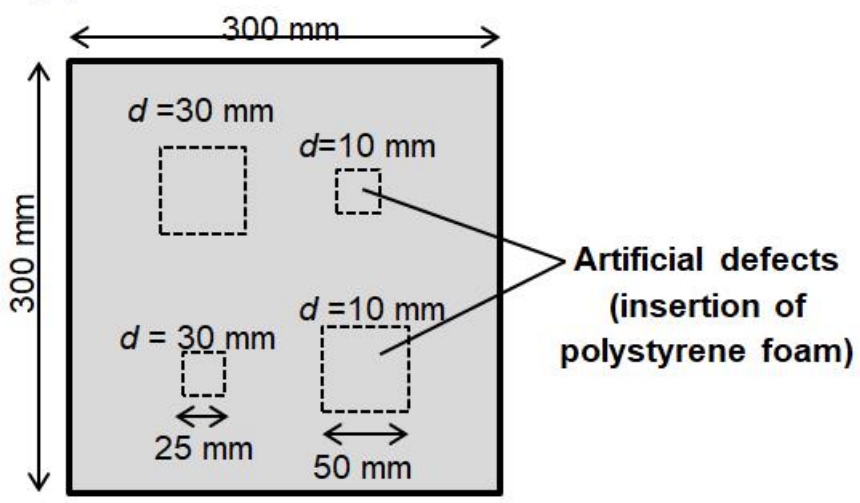

Fig. 2 (a) Photograph, and (b) Schematic illustration of concrete specimen used in experiment.

\subsubsection{Using a $\mathrm{CO}_{2}$ laser heating system}

Schematic illustration of the experiment using laser heating is shown in fig.3. $\mathrm{A} \mathrm{CO}_{2}$ laser source (Multiscan VS, Rofin-Baasel Inc.) was used to heat the concrete specimen (same as in Fig. 2) located at $10 \mathrm{~m}$ distance from the laser head. The excited laser was focused to a spot $147 \mathrm{~mm}$ away from the laser head and the beam diameter was expanded beyond the focal point. The diameter of the laser beam at $10 \mathrm{~m}$ was approximately $400 \mathrm{~mm}$, which covered the entire area of the specimen surface. The laser irradiation density on the surface was approximately $700 \mathrm{~W} / \mathrm{m}^{2}$. 


\subsection{Experimental results}

Thermal images obtained using the halogen lamp and laser source are presented in Figs. 4 and 5 , respectively. In both images, the artificial defects were identified as local hotspots. Temperature contrast $(\Delta T)$ between defective and non-defective areas on the defect with depth of $10 \mathrm{~mm}(50 \times 50 \mathrm{~mm})$ as obtained from the lamp and laser heating data are plotted in Figs. 4(b) and 5(b), respectively. There was little difference between the temperature contrast values obtained by the two heating methods. The results show that both heating methods are effective in inspection of concrete located far distance, and that the inspection capability is almost same when heat input is matched.

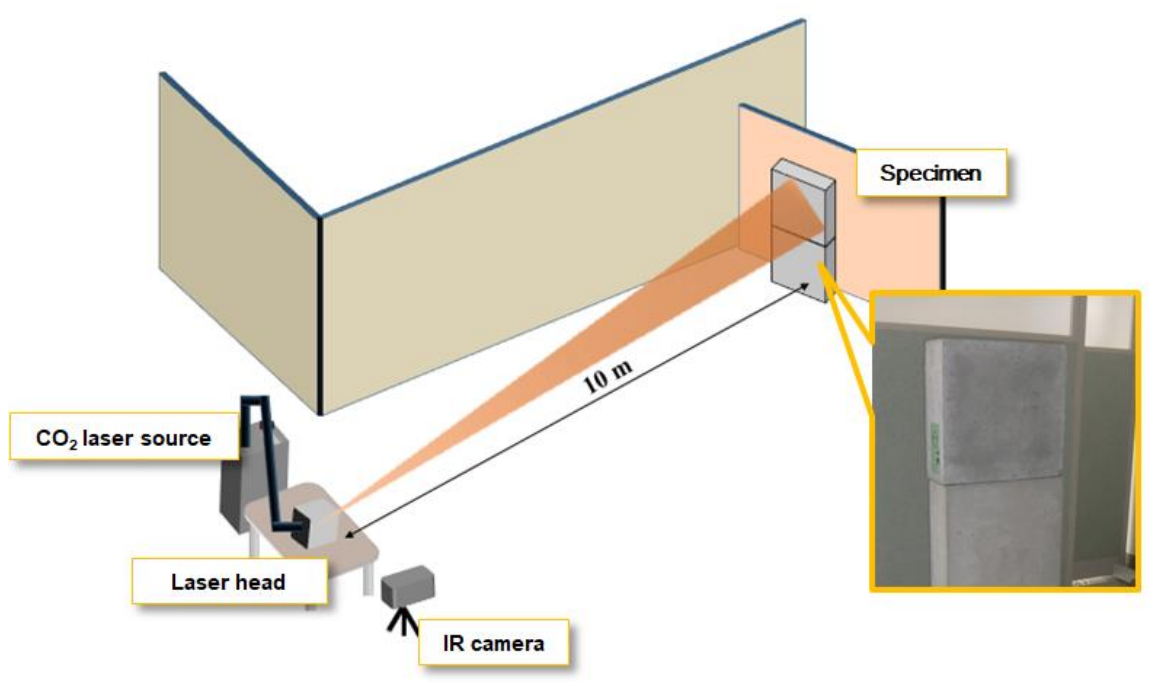

Fig. 3 Schematic illustration of experimental setup using $\mathrm{CO}_{2}$ laser source.

(a)
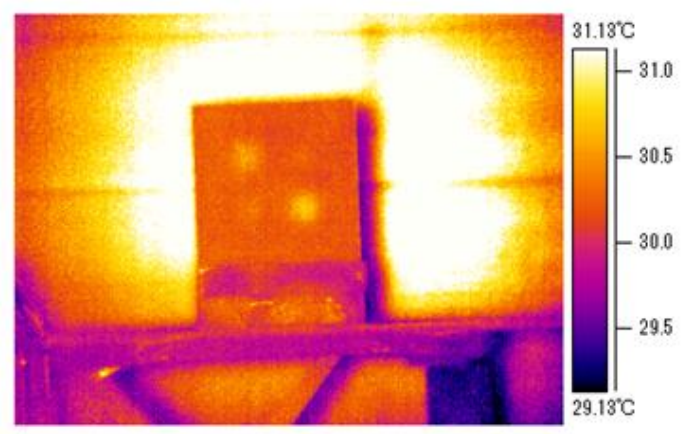

(b)

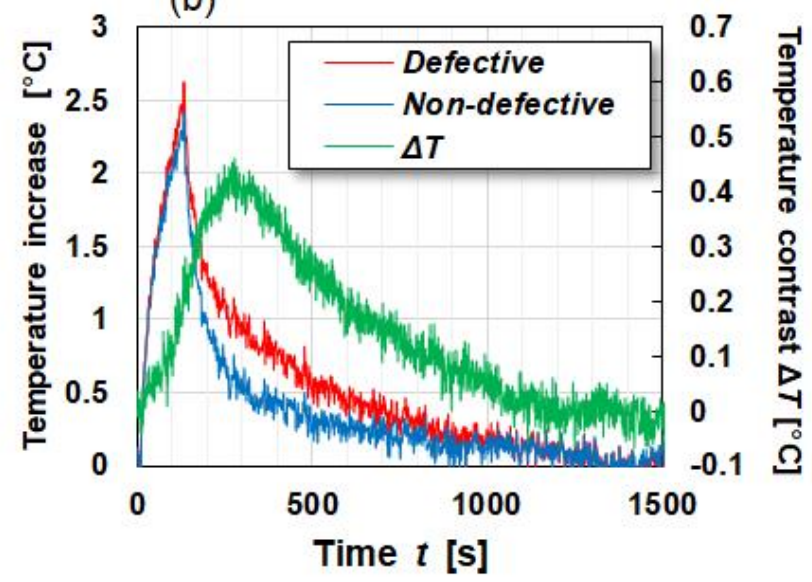

Fig. 4 Experimental results obtained using halogen lamp heating: (a) thermal image at 300 s, and (b) observed temperature increase and temperature contrast $\Delta T$ for defect of depth of $10 \mathrm{~mm}(50 \times 50 \mathrm{~mm})$. 
(a)

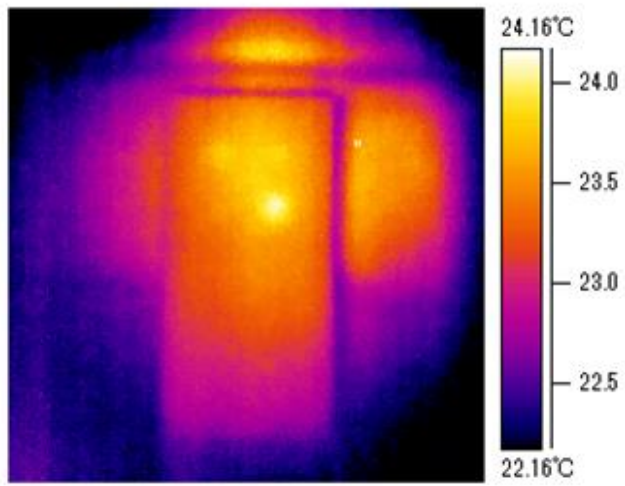

(b)

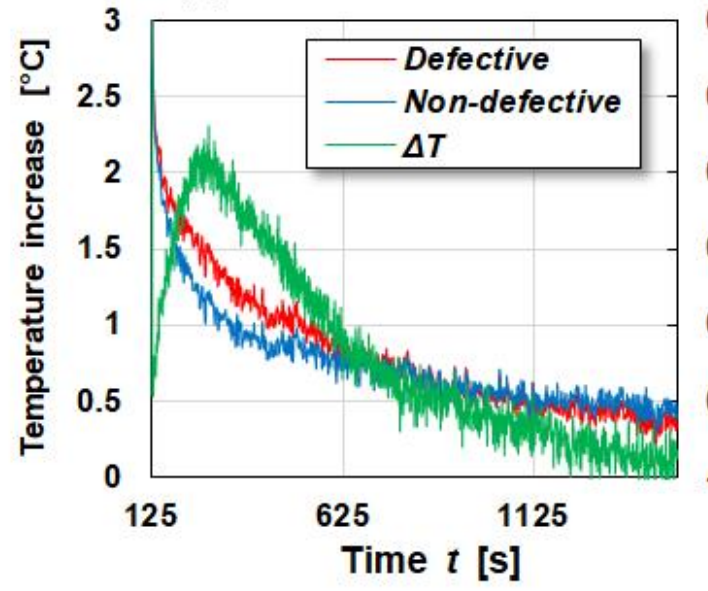

0.5

0.4

0.3

0.2

0.1

0

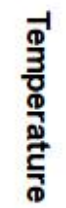

올

Fig. 5 Experimental results obtained using $\mathrm{CO}_{2}$ laser heating: (a) thermal image at $300 \mathrm{~s}$, and (b) observed temperature increase and temperature contrast $\Delta T$ for defect with depth of $10 \mathrm{~mm}(50 \times 50 \mathrm{~mm})$.

\section{Influence of surface discoloration of concrete}

Concrete surfaces are frequently discolored due to various factors. For example, the color becomes white when efflorescence occurs [3]. In thermographic inspections, such surface discoloration could adversely affect the inspection capability. This necessitated an investigation of the influence of discoloration on the measured inspection data using the halogen lamp and laser heating methods. In this experiment, aqueous calcium hydroxide solution was applied on the same specimen used in the previous study in order to simulate the discoloration caused by efflorescence. The discolored specimen is shown in Fig. 6.

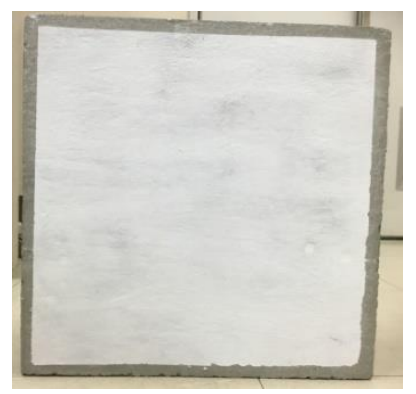

Fig. 6 Specimen with simulated discoloration, prepared by applying $\mathrm{Ca}(\mathrm{OH})_{2}$ solution to concrete surface (shown in Fig. 2).

The specimens were then heated by the halogen lamp and the $\mathrm{CO}_{2}$ laser from a distance of $10 \mathrm{~m}$, and surface temperature distribution during and after heating was monitored using infrared camera. Thermal images obtained from the simulated specimens using halogen lamp and laser heating are presented in Fig. 7, and the observed temperature contrast in the $10 \mathrm{~mm}$ deep defect $(50 \times 50 \mathrm{~mm})$ is plotted in Fig. 8 along with the temperature contrast obtained from a specimen without discoloration for comparison. It is clearly evident from Fig. 8 (a) that the temperature contrast obtained for the discolored specimen was significantly lower than that for the uncolored specimen in the case of halogen lamp heating. On the other hand, the difference in the temperature contrast between the discolored specimen and the uncolored specimen was less pronounced when $\mathrm{CO}_{2}$ laser source was used (Fig. 8 (b)). While using a halogen lamp, a large amount of light is likely to be reflected at the discolored surface thus lowering the heat input onto the specimen surface. This was further examined by the spectral characteristics of the specimen surface subject to the light irradiation. Figure 9 show the surface spectral reflectivity of the specimens (with and without discoloration) measured using a visible/near-infrared spectrophotometer (FieldSpec Pro, Analytical Spectral Devices Inc.). It is seen in Fig. 9 that the reflectance of the specimen with discoloration is higher (as much as two-fold) than that of the specimen without the discoloration at the wavelength of around $1000 \mathrm{~nm}$ (which is approximately the peak spectral intensity of the halogen lamp). The wavelength of the $\mathrm{CO}_{2}$ laser is $10600 \mathrm{~nm}$ and, as seen in Fig. 9, the difference between the reflectance decreases towards long wavelengths. This implies that the laser heating method would be more tolerant to influences 
arising from surface discoloration than the halogen lamp heating method. This would prove to be a major advantage of the laser heating method for inspecting concrete structures.

(a)

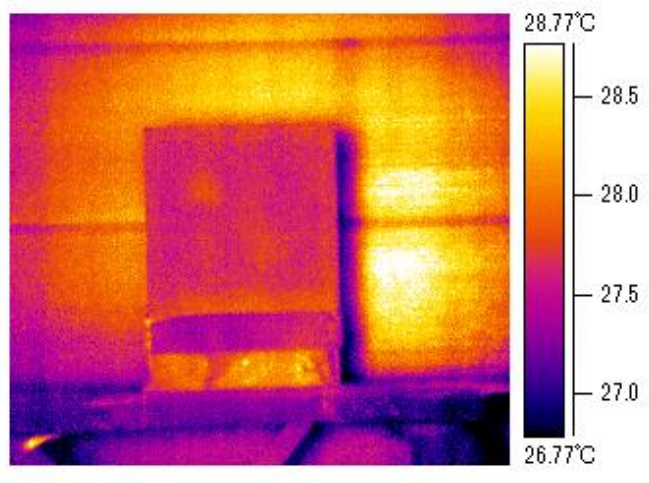

(b)

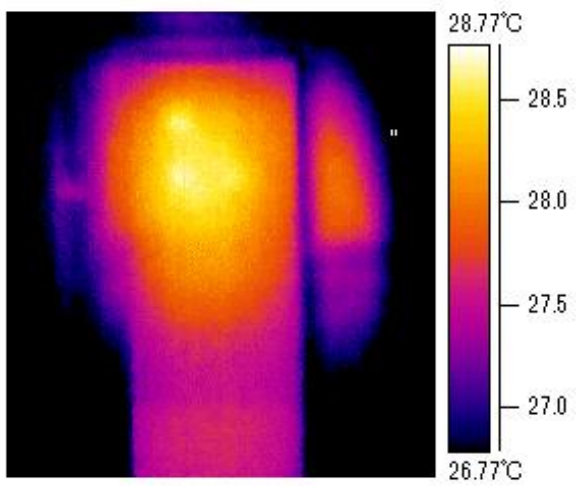

Fig. 7 Thermal images for specimen with discoloration obtained using (a) halogen lamp heating, and (b) $\mathrm{CO}_{2}$ laser heating. Both are thermal images at $300 \mathrm{~s}$ from start heating.

(a)

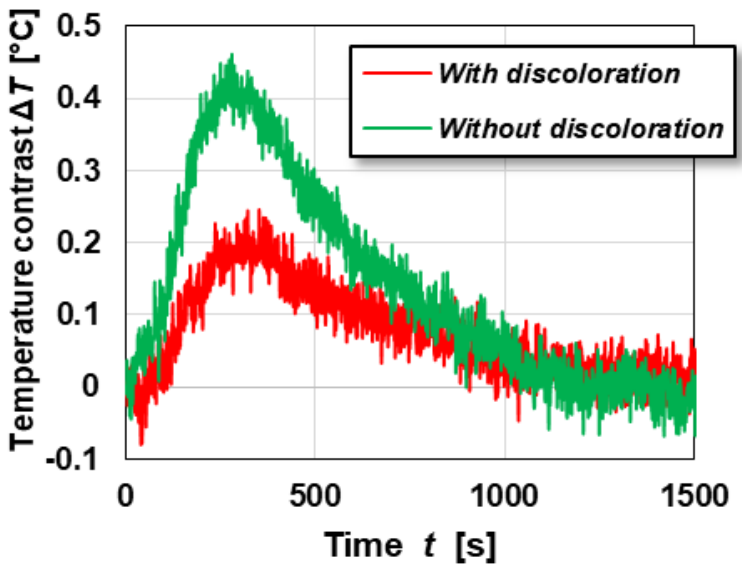

(b)

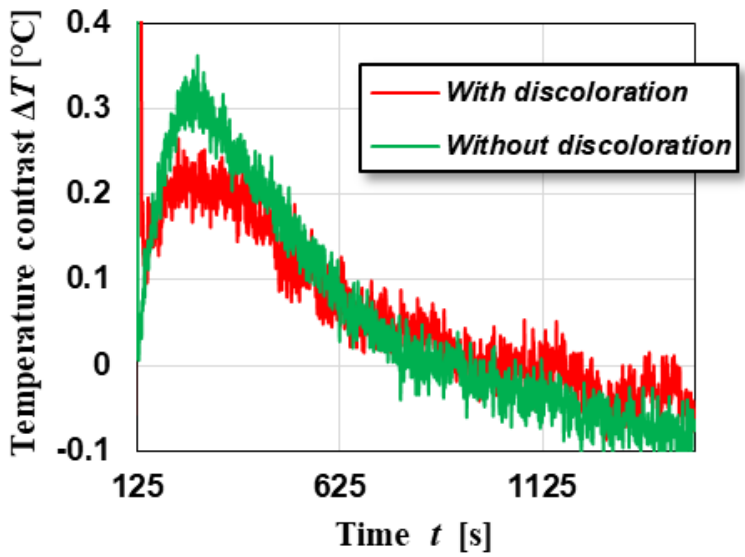

Fig. 8 Comparison of temperature contrast $\Delta T$ for defect with depth of $10 \mathrm{~mm}(50 \times 50 \mathrm{~mm})$ in specimen with and without discoloration obtained using (a) halogen lamp heating, and (b) $\mathrm{CO}_{2}$ laser heating.

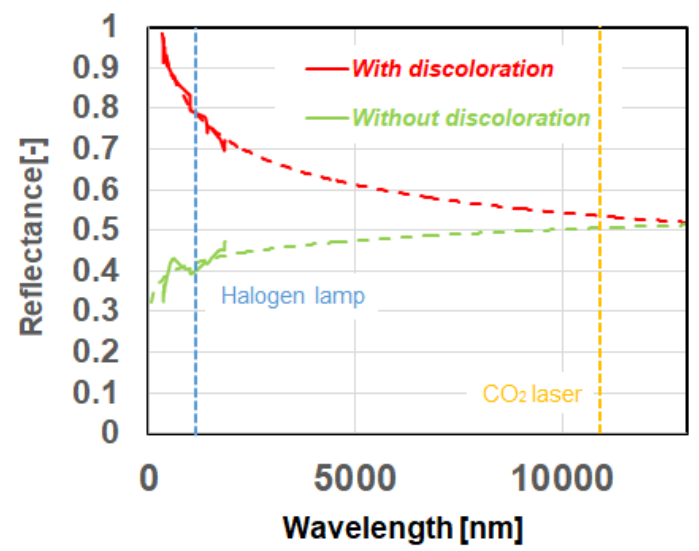

Fig. 9 Surface spectral reflectance for specimens, with and without discoloration. 


\section{Transformation into phase images}

In thermographic inspection, thermal images are sometimes transformed into phase images by applying Fourier transformation in order to enhance defect detectability; the technique is known as pulse phase thermography (PPT) when the test objects are heated by an instantaneous flash lamp [4,5]. The thermal images obtained in the present study were also analyzed by transforming them into phase images. The phase images thus obtained from the halogen lamp and laser heating experiments (in the process to obtain the phase images, Fourier transformation was applied to thermal data only after heating) are presented in Fig. 10. The darker spots seen are distinctly identified as defect centers in the concrete specimens, which clearly illustrate the profound detection capability of phase imaging in comparison with thermal imaging (Fig. 4(a) and Fig. 5(a)). Therefore, these results suggest that phase imaging in conjunction with lamp/laser remote heating is an effective inspection tool for concrete objects located at a distance.

(a)

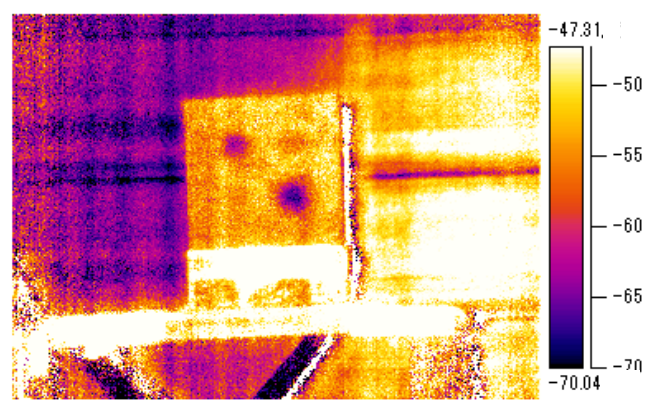

(b)

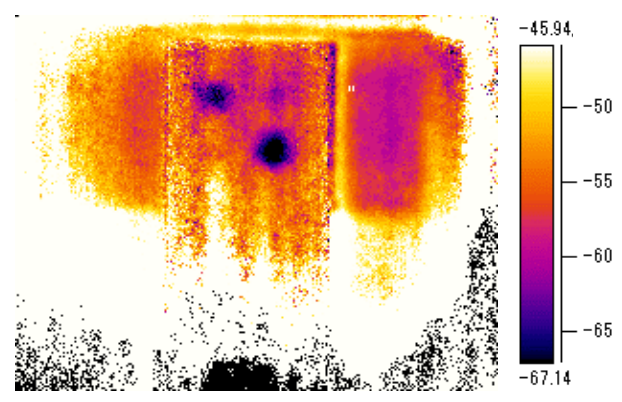

Fig. 10 Phase images at frequency of $0.0014 \mathrm{~Hz}$ transformed from thermal data obtained using (a) halogen lamp heating, and (b) $\mathrm{CO}_{2}$ laser heating.

\section{Conclusions}

Active thermographic inspection of concretes located at a distance was undertaken using two different remote heating apparatuses. It was ascertained from separate experiments that there is little difference between the inspection capability of optical halogen lamp-mirror method and $\mathrm{CO}_{2}$ laser heating when the heat input of both sources were matched. By employing Fourier transformation to generate phase images, the defect detectability of both heating methods was improved. While inspecting concrete surfaces which were discolored due to efflorescence, defect detection was relatively easier with $\mathrm{CO}_{2}$ laser heating method than with the halogen lamp due to higher reflectance of the latter. This emerged as an advantage of using laser heating. In addition, the laser method would be able to transfer heat to more distant objects by controlling the focal distance of the laser beam. However, the use of high-power laser apparatus must be controlled due to safety concerns. This is a factor that limits the convenience and usage of laser technique for routine inspections. In contrast, the halogen lamp heating can be used more conveniently, which is considered an advantage of using halogen lamp with light focusing apparatus.

\section{Acknowledgements}

This work was supported by JSPS KAKENHI Grant Number 17 K01296 and the MIKIYA Science and Technology Foundation.

\section{REFERENCES}

[1] D. Bates, at al., "Rapid thermal non-destructive testing of aircraft components", Composites Part B: Engineering, vol. 31, no. 3 pp. 175-185, 2000.

[2] N. Ogasawara, et al., "Paint delamination inspection for light buoys by infrared thermography with distance heating", Advanced Experimental Mechanics, vol. 1, pp. 167-172, 2016.

[3] H. Brocken, and T. G. Nijland, "White efflorescence on brick masonry and concrete masonry blocks, with special emphasis on sulfate efflorescence on concrete blocks", Construction and Building Materials, vol. 18, no. 5, pp. 315-323, 2004.

[4] X. Malague, and S. Marinetti, "Pulse phase infrared thermography", Journal of applied physics, vol. 79, no. 5, pp. 2694-2698, 1996.

[5] X. Maldague, Y. Largouët, and J. P. Couturier, "A study of defect depth using neural networks in pulsed phase thermography: modelling, noise, experiments", Revue générale de thermique, vol. 37, no. 8, pp. 704-717, 1998. 